Full Length Article

\title{
The formation of a Sn monolayer on Ge(100) studied at the atomic scale
}

\author{
Emily V.S. Hofmann ${ }^{\text {a,b,c, }}$, Emilio Scalise ${ }^{\mathrm{d}}$, Francesco Montalenti ${ }^{\mathrm{d}}$, Taylor J.Z. Stock ${ }^{\mathrm{b}}$, \\ Steven R. Schofield ${ }^{\text {b, }}$, Giovanni Capellini ${ }^{\mathrm{a}, \mathrm{f}}$, Leo Miglio ${ }^{\mathrm{d}}$, Neil J. Curson ${ }^{\mathrm{b}, \mathrm{c}}$, Wolfgang \\ M. Klesse ${ }^{\text {a, }}$
}

${ }^{a}$ IHP - Leibniz-Institut für Innovative Mikroelektronik, Im Technologiepark 25, D-15236 Frankfurt (Oder), Germany

${ }^{\mathrm{b}}$ London Centre for Nanotechnology, University College London, 17-19 Gordon Street, London WC1H OAH, UK

${ }^{\mathrm{c}}$ Department of Electronic and Electrical Engineering, University College London, London WC1E 7JE, UK

${ }^{\mathrm{d}}$ L-NESS and Dipartimento di Scienza dei Materiali, Università di Milano-Bicocca, Via R. Cozzi 55, I-20125 Milano, Italy

${ }^{\mathrm{e}}$ Department of Physics and Astronomy, University College London, London WC1E 6BT, UK

${ }^{\mathrm{f}}$ Dipartimento di Scienze, Università Roma Tre, V.le G. Marconi 446, I-00146 Rome, Italy

\section{A R T I C L E I N F O}

\section{Keywords:}

STM

DFT

GeSn

Wetting layer

\begin{abstract}
A B S T R A C T
The growth of multi-layer germanium-tin (GeSn) quantum wells offers an intriguing pathway towards the integration of lasers in a CMOS platform. An important step in growing high quality quantum well interfaces is the formation of an initial wetting layer. However, key atomic-scale details of this process have not previously been discussed. We use scanning tunneling microscopy combined with density functional theory to study the deposition of $\mathrm{Sn}$ on $\mathrm{Ge}(100)$ at room temperature over a coverage range of 0.01 to 1.24 monolayers. We demonstrate the formation of a sub- $2 \%$ Ge content GeSn wetting layer from three atomic-scale characteristic addimer structural components, and show that small quantities of Sn incorporate into the Ge surface forming two atomic configurations. The ratio of the ad-dimer structures changes with increasing Sn coverage, indicating a change in growth kinetics. At sub-monolayer coverage, the least densely packing ad-dimer structure is most abundant. As the layer closes, forming a two-dimensional wetting layer, the more densely packing ad-dimer structure become dominant. These results demonstrate the capability to form an atomically smooth wetting layer at room temperature, and provide critical atomic-scale insights for the optimization of growth processes of GeSn multi-quantum-wells to meet the quality requirements of optical GeSn-based devices.
\end{abstract}

\section{Introduction}

Germanium-tin (GeSn) alloys are the only group-IV semiconductors that demonstrate a direct band gap experimentally, expanding the reach of group-IV materials in both electronics [1] and photonics [2,3]. The first conclusive proof that the incorporation of $\mathrm{Sn}$ atoms into the $\mathrm{Ge}$ matrix leads to an indirect-direct band gap transition was presented by Wirths et al. who observed a change from an indirect to a direct gap in GeSn above $\sim 12$ at.\% Sn content [4]. Furthermore, recent advances in chemical vapor deposition (CVD) and molecular beam epitaxy (MBE) techniques enabled the epitaxial growth of high-quality GeSn on silicon (Si) substrates by using optimized Ge based buffers $[5,6]$. Consequently, GeSn alloys have generated increased research efforts towards their application in novel optoelectronic devices compatible with Si-based complementary metal-oxidesemiconductor (CMOS) technology.
A prominent example in the field of Si-photonics is the recent demonstration of lasing in GeSn alloys with Sn contents between 15\% and $20 \%$ at temperatures up to $270 \mathrm{~K}[7,8]$. A promising pathway towards this end is a multi-quantum-well (MQW) fabrication approach from the early $1990 \mathrm{~s}$, applied to GeSn-based heterostructures [9], as light emission is more efficient from lower dimensional structures [10]. Following this route P. Vogl et al. deposited a few monolayers (ML) of Sn and subsequently overgrew with tens of ML of Ge in alternating steps to form a super-lattice exhibiting a narrow direct bandgap [11]. The Sn layers were observed to only remain atomically flat when a thickness of 2.2 ML is not exceeded and intermixing of $\mathrm{Sn}$ and Ge to occur during Ge overgrowth. Overall, this presents a promising avenue for the fabrication of very thin, high Sn content GeSn quantum-wells in a Ge matrix $[9,12,13]$.

Beyond optoelectronic applications, GeSn/Ge MQW structures are of

\footnotetext{
* Corresponding authors.

E-mail addresses: emily.hofmann.16@ucl.ac.uk (E.V.S. Hofmann), klesse@ihp-microelectronics.com (W.M. Klesse).
} 
interest in the emerging Ge platform for quantum computing due to the large spin-orbit coupling in Sn $[14,15]$. Furthermore, few-layer stanene (graphene-like $\mathrm{Sn}$ ) grown on PbTe shows superconductivity below $2 \mathrm{~K}$ [16]. Grown on $\mathrm{Ge}(111)$, stanene is predicted to be a room temperature quantum spin hall insulator [17], which is of interest for low loss, timereversal protected electron transport.

However, despite the multitude of GeSn alloy and Sn thin-film activities, research in $\mathrm{Sn} / \mathrm{Ge}$ and GeSn surface properties remains scarce. In particular, the initial stages of physical vapor deposition Sn growth on the Ge surface are not well understood at the atomic scale. Scanning tunneling microscopy (STM) is uniquely suited to determining the electronic and structural properties of the surface at this scale, as it probes the local density of states at the surface. The study of initial Sn growth on $\mathrm{Ge}(100)$ after sub-monolayer Sn deposition at room temperature reveals small clusters forming $[18,19]$. In particular, Tomatsu et al. observed three ad-dimer configurations (A to C) after Sn deposition below 0.1 ML [19]. They found two types of ad-dimers that are located between dimer rows and orient parallel to the substrate dimers. The A features are symmetric and $\mathrm{A}$ feature chains align in the $\langle 310\rangle$ direction whereas the $\mathrm{B}$ features are tilted and chains align in the $\langle 110\rangle$ direction. They propose in analogy to the Ge on $\mathrm{Si}(100)$ system that the formation of a $\langle 110\rangle$ chain is energetically favored. However, a higher diffusion barrier for a $\mathrm{Sn}$ atom to locate in the position for the next feature in a $\langle 110\rangle$ chain, as opposed to a $\langle 310\rangle$ chain, may result in the $\langle 310\rangle$ chains being kinetically favored.

Beyond $\mathrm{A}$ and $\mathrm{B}$ features, a third ad-dimer configuration, the $\mathrm{C}$ feature, was also observed [19]. It is a tilted ad-dimer oriented perpendicular to the substrate dimers and located on the reaction sites for the growth of the next diamond-cubic layer. Additionally, a separate STM study found Ge-Sn heterodimers formed in the Ge(100) surface layer could exist in one of two buckling orientations [20]. However, the influence of the STM tip may reverse the buckling orientation of the Sn atom during imaging. In this work, we investigate the deposition of Sn on $\mathrm{Ge}(100)$ at room temperature and up to the formation of an abrupt and atomically flat Sn wetting layer using atomic-resolution STM and density functional theory (DFT) simulations.

In the first segment we extend the work of Tomatsu et al. $[19,20]$ on low Sn coverage ( 0.01 monolayer), and based on DFT modelling, we are able to confirm the four Sn-related surface features to be: surface incorporated $\mathrm{Sn}$ in the form of Ge-Sn heterodimers, and the three distinct $\mathrm{Sn}$ ad-dimer configurations suggested previously. Going beyond previous work, we clarify the chemical composition of the ad-dimers by detailed comparison between DFT simulations and experimental images. We conclusively show that the surface ad-dimers are not exclusively Sn, but some contain ejected Ge atoms. Thus, there exists a competition between stabilization mechanisms involving $\mathrm{Sn}$ surface agglomeration versus incorporation of $\mathrm{Sn}$ ad-atoms into the top $\mathrm{Ge}$ surface layer and consequent ejection of a Ge surface atoms on top of the surface. As this necessarily produces Sn atoms on different lattice planes, it introduces already at room temperature an inherent limitation on suppressing the intermixing at $\mathrm{Ge} / \mathrm{Sn}$ interfaces for $\mathrm{Ge} / \mathrm{Sn}$ quantum well structures or Sn quantum dots on Ge in future device applications.

In the second segment of this paper, we gradually increase the Sn coverage and investigate surfaces with up to and slightly beyond full monolayer coverage (1.24 ML). Using careful feature identification and counting we determine how the relative densities of these features evolve with increasing coverage, highlighting a change in formation kinetics. Overall, our combined STM and DFT surface study presents a key step towards a deeper understating of the underlying mechanisms governing early stages of Sn-on-Ge growth.

\section{Methods}

All STM samples were prepared from antimony doped Ge(100) wafers in ultra-high vacuum with a base pressure below $5 \times 10^{-10} \mathrm{mbar}$. New Ge substrates were degassed for a total of $3 \mathrm{~h}$ at $\sim 250{ }^{\circ} \mathrm{C}$ before being annealed up to $800{ }^{\circ} \mathrm{C}$ by direct current heating for $1 \mathrm{~h}$. The surface was then cleaned by repeated sputter-anneal cycles of argon (Ar), sputtering at $1 \times 10^{-5} \mathrm{mbar}, 800-1200 \mathrm{~V}$ accelerating voltage, and $10 \mathrm{~mA}$ emission current, and then subsequent annealing to $800^{\circ} \mathrm{C}$. Care was taken to cool samples at a rate no faster than $50^{\circ} \mathrm{C} / \mathrm{min}$ when above $500{ }^{\circ} \mathrm{C}$. To improve surface quality, epitaxial Ge could be grown by Knudsen cell evaporation for $1 \mathrm{~h}$ at $500{ }^{\circ} \mathrm{C}$. Finally, it was possible to remove $\mathrm{Sn}$ from the Ge surface by annealing up to $800{ }^{\circ} \mathrm{C}$ for $1 \mathrm{~h}$ and then flashing to $800{ }^{\circ} \mathrm{C}$.

Once a contamination-free $\mathrm{Ge}(100)$ surface with surface defect density below $1 \%$ was achieved, as verified by STM, Sn was deposited via e-beam evaporation onto the room temperature $\mathrm{Ge}(100)$ surface at a rate of $0.002 \mathrm{ML} / \mathrm{s}$. For this work, samples were prepared at four different area coverages: 0.01 ML, 0.05 ML, 0.53 ML, and 1.24 ML, then measured at room temperature in a SPECS SPM 150 Aarhus HT in STM mode. All scans were obtained with an electrochemically DC etched tungsten tip in constant current mode. STM images were recorded as the STM tip traced and retraced each horizontal line before progressing to the next vertical position, providing the possibility of obtaining dualbias scans where the bias for trace $(-1.5 \mathrm{~V})$ and retrace $(+1.0 \mathrm{~V})$ were different. Sn surface coverages were determined from STM images by height thresholding the ad-features against the substrate and determining the percentage of area above the threshold.

The surface features were further characterized by means of DFT simulations. DFT calculations were performed by exploiting PBE exchange correlation potentials [21] and projector augmented plane-wave bias sets with a 60 Ry kinetic energy cut-off, as implemented in the Quantum Espresso package [22]. From there, the Tersoff-Hamann approximation was used to produce simulated STM images at $0 \mathrm{~K}$. Therein, the tunneling current is proportional to the local density of states integrated from the Fermi-level of the sample to the applied bias. The values of the isosurface for the STM-image in constant current mode has been set to $0.5 \times 10^{-7} \mathrm{e} / \mathrm{a}_{0}^{3}$ and an applied bias of $-1.5 \mathrm{~V}$ and $+0.8 \mathrm{~V}$ simulated. These biases enable comparison of the simulations with experimental dual-bias scans.

\subsection{Surface features at sub-monolayer coverage}

As a first step towards understanding the formation of the wetting layer, we extend the interpretation of previously observed features at sub-monolayer coverages. At room temperature the clean $\mathrm{Ge}(100)$ surface (Fig. 1(a)) consists of dimers organized in rows. When buckled they form a $\mathrm{c}(4 \mathrm{x} 2)$ reconstruction whereas the dimers appearing unbuckled organize in a $\mathrm{p}(2 \mathrm{x} 1)$ reconstruction. The apparent lack of buckling in the $\mathrm{p}(2 \mathrm{x} 1)$ reconstruction is due the buckling orientation changing faster than the STM can detect. The $\mathrm{c}(4 \mathrm{x} 2)$ and $\mathrm{p}(2 \mathrm{x} 2)$ reconstructions are energetically similar, resulting in domains of both reconstructions on the surface, with their presence often stabilized by defects, adsorbates and step-edges [23].

The underlying structure of the substrate does not change when depositing 0.05 ML Sn on the surface, as demonstrated in Fig. 1(b). However, in agreement with previous literature [18-20], two categories of additional surface features appear: (i) Slight protrusions among the Ge substrate dimers (three examples are shown in the inset of Fig. 1(b)) and (ii) clusters of varying shapes and sizes, examples of which are shown in more detail in Fig. 1(c-f). Close examination of the surface clusters reveals that these comprise the three basic atomic-scale components, which were first described by Tomatsu et al. [19]. As shown in Fig. 1(c), the A feature is the darkest feature in filled STM images taken at $-1.5 \mathrm{~V}$, appearing as a double protrusion between two surface dimer rows. Note, directly attached neighboring A features align in the $\langle 310\rangle$ directions. In contrast, the $\mathrm{B}$ and $\mathrm{C}$ features are approximately equally bright in filled STM (at $-1.5 \mathrm{~V}$ ), and are only distinguishable by how they align to neighboring features of the same type and to the surface dimers. Neighboring B features align in rows perpendicular to the $\mathrm{Ge}$ (100) dimer rows ( $\langle 110\rangle$ direction), while $\mathrm{C}$ features align in a zig-zag 

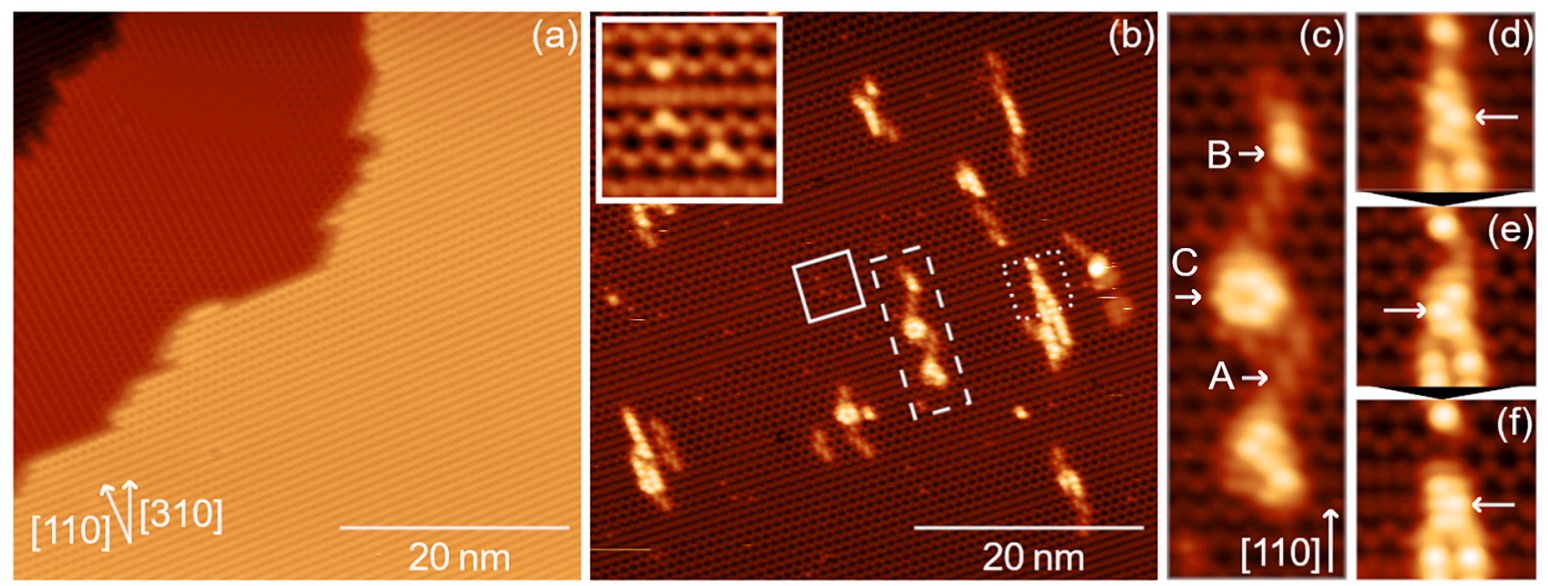

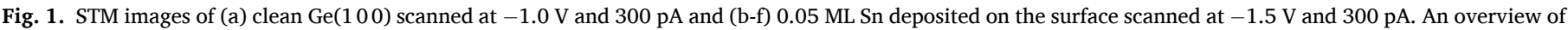

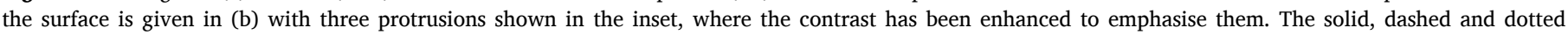

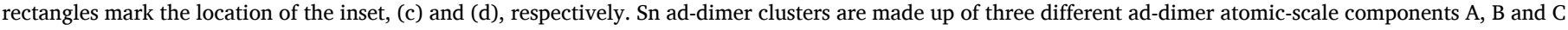

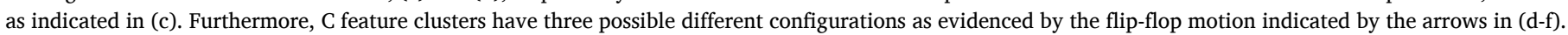

pattern oriented perpendicular to the surface dimers. Often, feature clusters will contain long chains of A or B features. It has been proposed [19] that the formation of these chains can be understood in analogy to Qin and Lagally's model of Ge on Si(100) [24].

Furthermore, in Fig. 1(d-f) we show the first observation of clusters of $\mathrm{C}$ features changing buckling orientation over periods of tens of minutes during STM imaging. In the first panel (Fig. 1(d)) the bright protrusions of the $\mathrm{C}$ features alternate position in the pattern left-right-left, with the arrow highlighting the middle protrusion. When the feature was next scanned 13 min later in Fig. 1(e) the orientation had flipped to right-left-right whereas $61 \mathrm{~min}$ later (Fig. 1(f)) the protrusions had smeared out indicating a symmetric dimer. Simultaneously, the buckling of the substrate dimers changed indicating strong ad-dimer-surface interactions.

To further understand the behavior of $\mathrm{Sn}$ atoms upon adsorption on $\mathrm{Ge}(100)$ we studied the above described Sn related surface features in more detail. We begin with the slight protrusions among the Ge surface atoms as in the inset of Fig. 1(b), which have been previously assigned to Ge-Sn heterodimers [19,20]. In Fig. 2(a-c) we show dual-bias scans (-1.5 $\mathrm{V}$ and $+1.0 \mathrm{~V}$ ) of three similar, but subtly different, features taken from a surface with $0.05 \mathrm{ML}$ area Sn coverage. Alongside each of the experimental STM images we show one of three different DFT simulated images as follows: a Ge-Sn heterodimer with an up-tilted Sn atom (Fig. 2 (a)), a Ge-Sn heterodimer with a down-tilted Sn atom (Fig. 2(b)), and a Ge-Ge-H hemihydride dimer (Fig. 2(c)). Note, that at temperatures approaching $0 \mathrm{~K}$ - the temperature of the DFT simulations - the Ge(1 000$)$ surface is completely $\mathrm{c}(4 \mathrm{x} 2)$ reconstructed, limiting the direct comparison to this surface reconstruction. Nonetheless, comparisons to analogous features on the high temperature $\mathrm{p}(2 \mathrm{x} 1)$ reconstruction can be extrapolated. We assign each of the experimentally observed features to the corresponding DFT simulated feature based on the closeness of appearance of the experimental and simulated image, and by consideration of their surface density as discussed below.

The most common feature, shown in Fig. 2(a), constitutes $93 \%$ of these three features, obtained from a total of 384 slight protrusions counted on an area of $22,500 \mathrm{~nm}^{2}$ covered in $0.05 \mathrm{ML}$ Sn. In our STM images it appears as a slight protrusion at the location of a single atom (the brightest atom in Fig. 2(a)) in both filled electronic states (negative bias) and, located on the other side of the dimer row, in empty electronic states (positive bias). In agreement with DFT simulations, we may assign this feature to a Ge-Sn heterodimer in the up-tilted position, as indicated in the schematic. In contrast, the down-tilted Ge-Sn heterodimer is the least common of these features with $<1 \%$ contribution to the slight protrusions in the surface. As can be seen in Fig. 2(b), it is indistinguishable from the Ge substrate in filled states while appearing similar to the up-tilted Ge-Sn heterodimer in empty states.

The observation of a much greater abundance of up-tilted Ge-Sn heterodimers is supported by DFT calculations showing that these features are $60 \mathrm{meV}$ more favorable than the down-tilted Ge-Sn heterodimers. This energy difference is small enough that the presence of down tilted Ge-Sn heterodimers may be induced by tip to sample energy transfer during scanning. This is consistent with the previous observation of a STM induced buckling orientation flip of a Ge dimer row containing the up-tilted Ge-Sn heterodimer [20].

Finally, we highlight that the remaining $7 \%$ of slight protrusions on the surface are not actually $\mathrm{Sn}$ related but instead appear to be Ge-Ge-H hemihydrides [25], where hydrogen ad-atoms are attached to the dangling bond of the lower Ge atom (see Fig. 2(c)). In filled states the hemihydride appears as a protruding atom similar to the up-tilted Ge-Sn heterodimer (compare Fig. 2(a) with Fig. 2(c)) but it is clearly distinct from the other two features in empty states, where it appears as a threedimer wide depression with a slight protrusion at the same location as in filled states. We note that, the presence of hemihydrides on the surface cannot be avoided, as they are a consequence of residual water and hydrogen in the UHV system.

Overall, we identified two types of Ge-Sn heterodimers, which indicates intermixing of $\mathrm{Sn}$ and $\mathrm{Ge}$ at the surface. The up-tilted and downtilted Ge-Sn heterodimer are distinguishable from each other in filled state STM. However, the unambiguous assignment of Ge-Sn heterodimers always requires both filled and empty state STM. In filled state STM alone, the down-tilted Ge-Sn heterodimers appear nearly identical to Ge-Ge surface dimers and the up-tilted Ge-Sn heterodimer similar to $\mathrm{H}$-induced surface features.

As a direct consequence of Sn ad-atoms partially incorporating into the top surface layer and forming the heterodimers, Ge must be ejected to sites on top of the surface. These ejected Ge atoms may either attach to step edges or incorporate into the ad-dimer clusters. Hence, to investigate the chemical composition of the ad-dimer clusters, which may contain both adsorbed $\mathrm{Sn}$ as well as ejected Ge ad-atoms, we compare in Fig. 3 the experimental dual-bias images of the basic atomic-scale components "A", "B", and "C" to DFT simulations of clusters containing only $\mathrm{Sn}-\mathrm{Sn}$ or Ge-Ge ad-dimers.

As previously mentioned, feature $\mathrm{A}$ is a symmetric ad-dimer oriented parallel to the substrate dimers, feature B is an out-of-plane tilted addimer oriented parallel to the substrate dimers, and feature $C$ is an out-of-plane tilted ad-dimer oriented perpendicular to the substrate dimers. Qualitatively the agreement between simulation and experimental image is excellent for all three ad-dimers independent of the chemical 


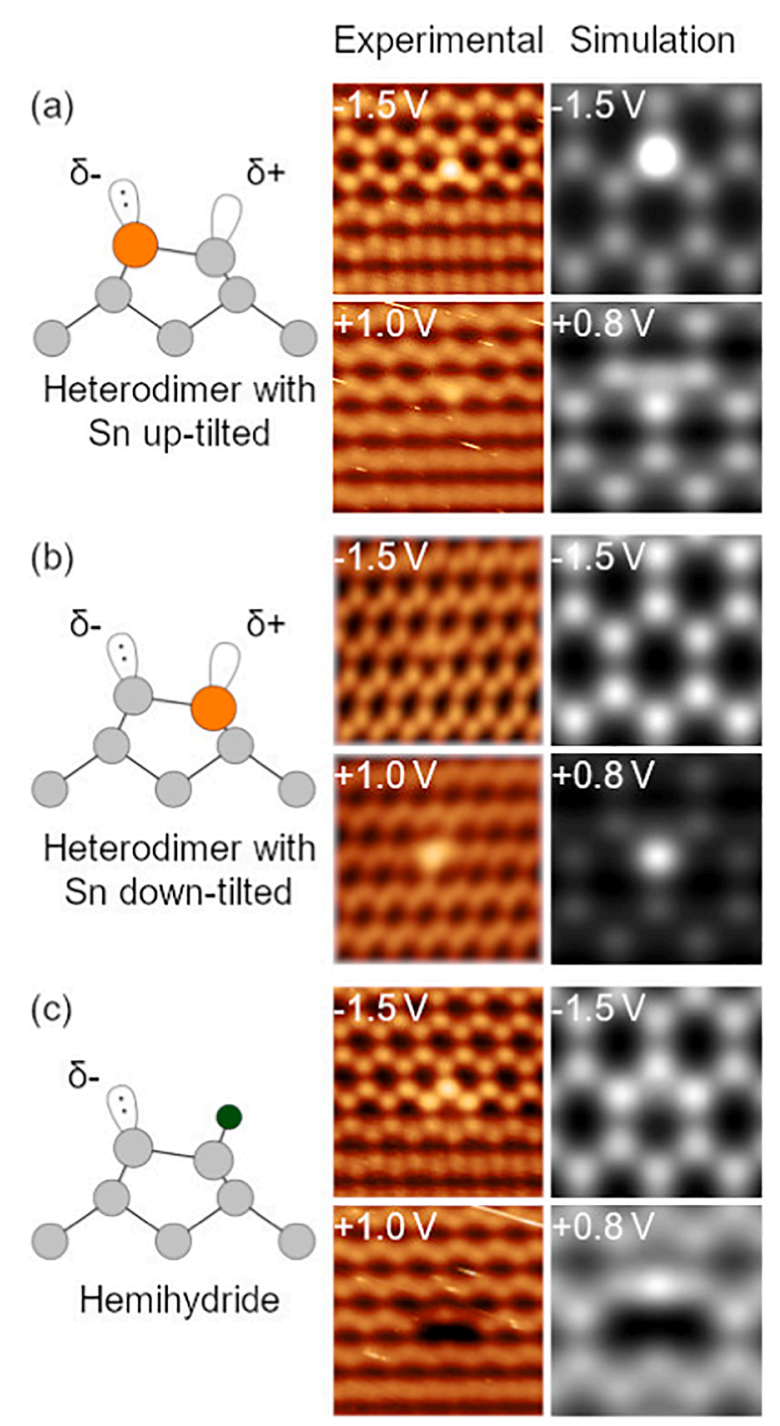

Fig. 2. The Ge-Sn heterodimer with $\mathrm{Sn}$ in the upper (a) and lower position (b) as well as the hemihydride (c). In the side view schematic of each feature, gray indicates $\mathrm{Ge}$ atoms, $\mathrm{Sn}$ is marked in orange, and hydrogen in green. STM data taken at $300 \mathrm{pA}$ and $-1.5 \mathrm{~V}$ or $+1.0 \mathrm{~V}$ is compared to DFT simulations for -1.5 $\mathrm{V}$ and $+0.8 \mathrm{~V}$. For all three features there is good agreement between the experimental data and the simulations. (For interpretation of the references to colour in this figure legend, the reader is referred to the web version of this article.)

species used in the simulation. Feature A (Fig. 3(a)) shows a slight double protrusion in filled states in experiment, and in both Ge-Ge and Sn-Sn dimer simulations. The corresponding empty states image for all three cases shows a single protrusion per dimer, brighter than the filled states double protrusion. In Fig. 3(b) the experimental panels show three $\mathrm{B}$ features at the center of the cluster (highlighted by the arrows in filled states), each exhibiting a single bright protrusion both in filled and empty states. The simulations of this feature as Ge-Ge and Sn-Sn dimers both reproduce that characteristic with no qualitative difference. Finally, the cluster of three $C$ features in the experimental panels of Fig. 3(c) shows three distinct protrusions alternating from right to left to right in filled states imaging. In empty states the individual protrusions are less distinct and alternate from left to right to left. This appearance is reproduced in the corresponding DFT simulations of both the Ge-Ge dimer and $\mathrm{Sn}$-Sn ad-dimers. In both cases there are three distinct protrusions alternating position in filled states and less distinct protrusions on the other side of the feature in empty states. Therefore, from this qualitative comparison of experimental and simulated STM images the structure of the ad-dimers can be confirmed but not the chemical configuration.

As a next step we attempt to quantify the difference between Ge-Ge dimers and Sn-Sn dimers by extracting the height difference between the maximum of a Ge substrate dimer and the maximum of an ad-dimer from the DFT simulations of the surface under negative bias (Table 1). For all three atomic-scale component simulations the Sn-Sn ad-dimer is found to be higher than the Ge-Ge ad-dimer by several tenths of an Angstrom. While this is within the height resolution of STM measurements, the varying tilt angle of the Ge surface dimers at room temperature and the small size of the features under investigation do not allow quantitative experimental confirmation of the presence of both types of dimers, as any observed height differences in the STM images lie within the measurement uncertainty.

Nonetheless, in empty states STM images for all three atomic-scale components, A, B, and C surface clusters can be found where there is a clear brightness contrast between neighboring features of the same atomic configuration. One such example for a cluster of $C$ features, arranged similar to the Ge substrate $\mathrm{c}(4 \mathrm{x} 2)$ reconstruction, is shown in Fig. 4. The experimental panels show that there is no contrast difference in filled states, but in empty states the three $\mathrm{C}$ features on the left appear less bright than the three $C$ features on the right. When simulating the features as either pure Ge-Ge dimers (second row) or pure Sn-Sn dimers (third row) no contrast difference arises between the different locations of the $\mathrm{C}$ features. However, when replacing the lower atoms of the left dimers with $\mathrm{Ge}$ atoms with the remainder being Sn (bottom row) a contrast is produced in empty states but not in filled states. Therefore, this is a possible atomic configuration for the experimentally observed structure shown here. The comparison of the three simulations demonstrates that a single atomic species cannot produce a brightness contrast in features of the same type while mixed features containing both Ge and Sn can.

Overall we may conclude, that based on contrast differences within basic atomic-scale components $\mathrm{A}, \mathrm{B}$, and $\mathrm{C}$ we are able to demonstrate the presence of ad-dimers containing both $\mathrm{Ge}$ and $\mathrm{Sn}$ atoms, due to the surface incorporation of $\mathrm{Sn}$ ad-atoms and concomitant ejection of $\mathrm{Ge}$ surface atoms - even at room temperature.

\subsection{Evolution from low coverages to full monolayer coverages}

Driven by the technological applications associated with GeSn MQWs in the field of group-IV photonics, a detailed understanding of the wetting layer formation upon Sn deposition on Ge is of particular interest to ensure sharp interfaces [10]. Hence, to detail the gradual increase from sub- to full monolayer coverages we here leverage the atomic-scale imaging capabilities of high resolution STM and present the structural composition of the Ge surface after the RT deposition of $0.01 \mathrm{ML}, 0.05 \mathrm{ML}, 0.53 \mathrm{ML}$, and 1.24 ML of Sn (see Fig. 5).

At sub-monolayer coverages up to $0.53 \mathrm{ML}$, Fig. 5(a-c), the features discussed previously (i.e., A, B, C and heterodimer) are discernible on the surface, forming clusters of ad-dimers. As the Sn coverage increases these clusters gradually begin to cover the surface by elongating perpendicular to the dimer rows, with minimal growth along the dimer row direction. It has been proposed that the formation kinetics of such isolated 2D-chains of Sn ad-dimers is influenced by the surface strain induced by $\mathrm{Sn}$ adsorbates, with the strain playing a crucial role in the diffusion barrier height of Sn ad-atoms [19].

Moving to the monolayer regime, a filled state STM image of the surface after the RT deposition of 1.24 ML is shown in Fig. 5(d). Highresolution STM reveals the formation of a closed and atomically flat monolayer (Layer 1), even at RT. Although no long-range ordering is observed, the previously described features A, B, and C, appear to exclusively make up this wetting layer and smaller patches consisting of only one feature type can be observed, and are highlighted in Fig. 5(d). Note that regions of A features appears less bright than the geometrically similar B and C features, consistent with equivalent low coverage filled 
(a)

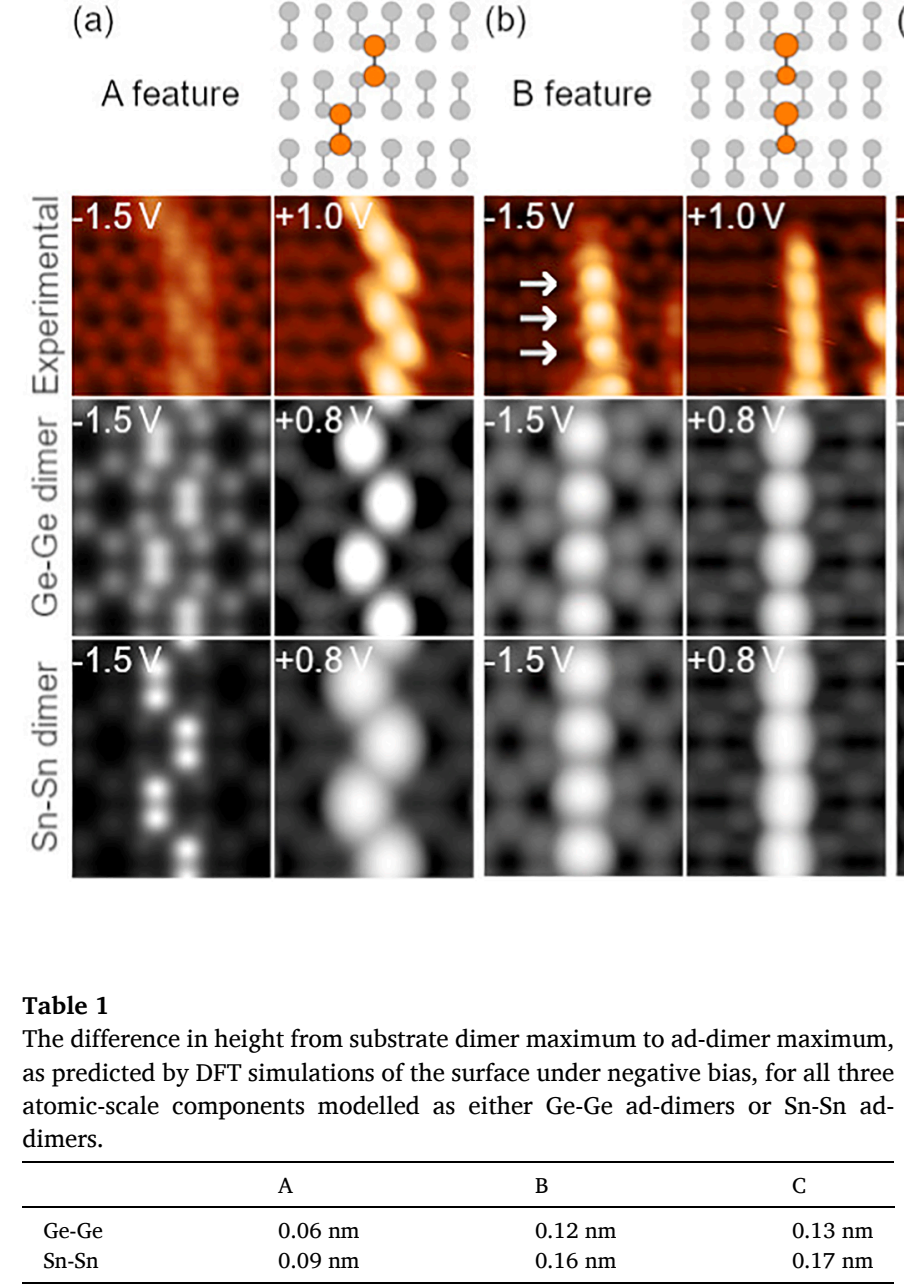

state STM data of Fig. 3. Consequently, the patches of A features in Fig. 5 (d) appear as holes in the wetting layer despite the surface being in fact completely covered. However, as shown in the magnified STM image in Fig. 5(e) taken in dual bias mode, the Ge substrate is occasionally visible in the vicinity of the A features, due to their low packing density allowing for large gaps that cannot be filled by further ad-dimers, which becomes particularly evident in the empty states image.

Furthermore, the STM images in Fig. 5(d) and (e) reveal the onset of formation of a second and a third layer consisting of new Sn-related surface features, whose study goes beyond the scope of this work. Despite the presence of individual features belonging to the third layer, no overall clustering was observed. In summary, at these coverages the first layer is complete and forms a wetting layer with patches of A, B and $\mathrm{C}$ features displaying short range order, but no long range order across the entire surface.

Next, the formation of the wetting layer was further studied by considering the evolution of features as coverage increases (Fig. 5(f)). For STM images obtained at each surface coverage, the number of atoms of each feature type were counted, and then normalized to a $2500 \mathrm{~nm}^{2}$ area of reactive sites. The uncertainties specified arise from averaging several STM measurements at different locations of the same sample. At an area coverage of $0.01 \mathrm{ML}$, feature $\mathrm{A}$ is most abundant followed by the heterodimer and then features B and C. However, while all four individual feature counts increase with coverage, the heterodimer quickly becomes the least abundant feature and the A feature count drops below first the $\mathrm{B}$ feature, and then the $\mathrm{C}$ feature as coverage increases above 0.5 ML. Therefore, in the completed wetting layer the B feature is most abundant at $44 \%$, closely followed by the $\mathrm{C}$ feature at $37 \%$, whereas the (c)

C feature

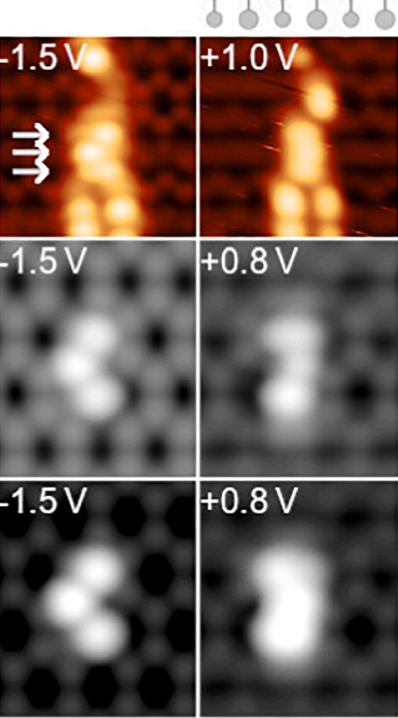

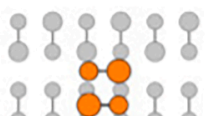

Fig. 3. The three ad-dimer features. Feature A (a) is a symmetric ad-dimer parallel to the substrate dimers as indicated in the schematic by the orange ad-dimers. Substrate dimers are marked in gray. In contrast B (b) and $C$ (c) are tilted dimers (the lower addimer atom is indicated by being drawn smaller) with the B features orienting parallel and the $C$ features perpendicular to the substrate dimers. STM scans (second row) taken at $300 \mathrm{pA}$ and $-1.5 \mathrm{~V}$ and $+1.0 \mathrm{~V}$ are compared to DFT simulations of a Ge-Ge addimer (third row) and a Sn-Sn ad-dimer (bottom row). In the STM image of (b) and (c) only the middle three features are B or C features, respectively, as highlighted by the arrows. In the simulations, four dimers are simulated for the A and B features, and three dimers for the $\mathrm{C}$ feature. (For interpretation of the references to colour in this figure legend, the reader is referred to the web version of this article.)

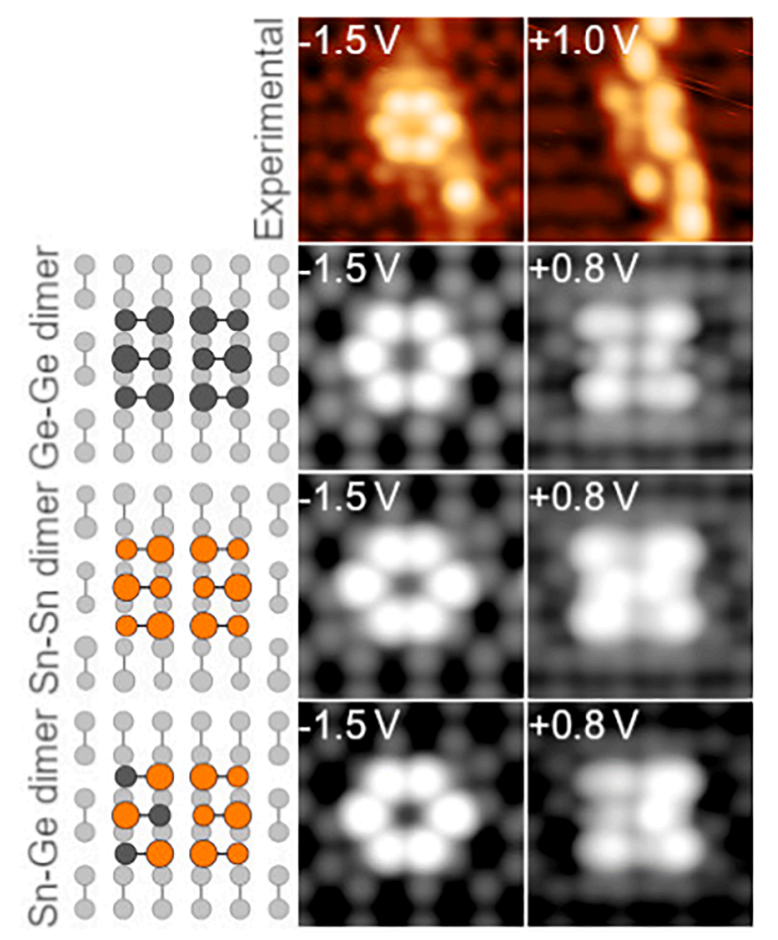

Fig. 4. A cluster of six $C$ features. The top row shows a dual-bias scan taken at 300pA tunnelling current. The simulations below are for the configurations shown on the left. Ge-Ge ad-dimers only (black atoms) in the second row, Sn-Sn ad-dimers only (orange atoms) in the third row and predominantly Sn atoms, but Ge atoms in the lower position of the dimer for the left three ad-dimers in the bottom row. The mixed feature shows the closest resemblance to the experimental data. (For interpretation of the references to colour in this figure legend, the reader is referred to the web version of this article.)

A feature only makes up $19 \%$ of the surface.

Next, from the structural schematics in Fig. 3 and the STM data in Fig. 5(e) it is discernible that the A feature packs least densely on the 
(a) $0.01 \mathrm{ML},-1.5 \mathrm{~V}$

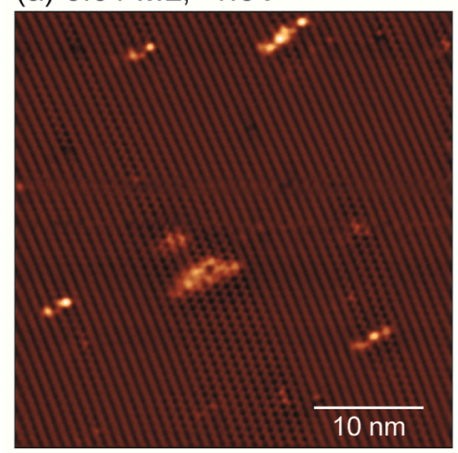

(b) $0.05 \mathrm{ML},-1.5 \mathrm{~V}$

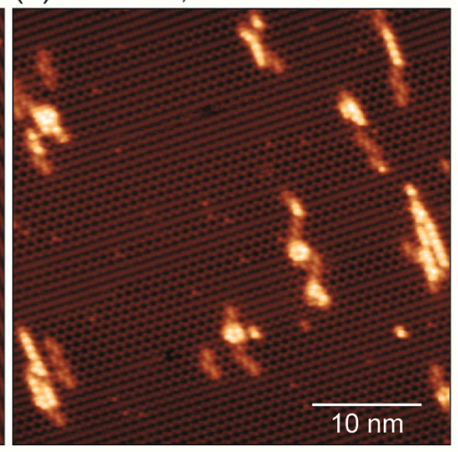

(c) $0.53 \mathrm{ML},-2.0 \mathrm{~V}$

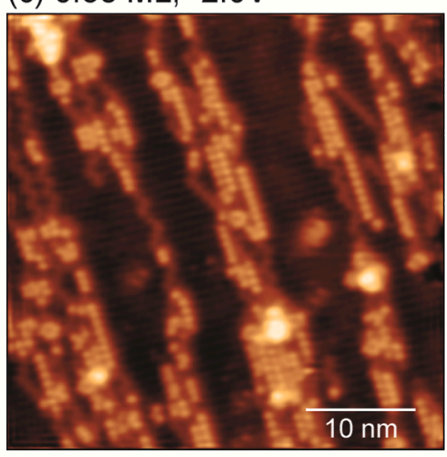

(d) $1.24 \mathrm{ML},-1.5 \mathrm{~V}$

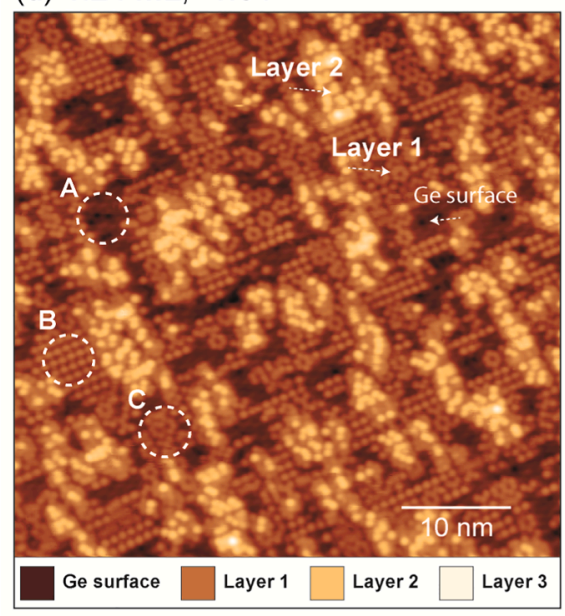

(e) $1.24 \mathrm{ML}$, dual bias

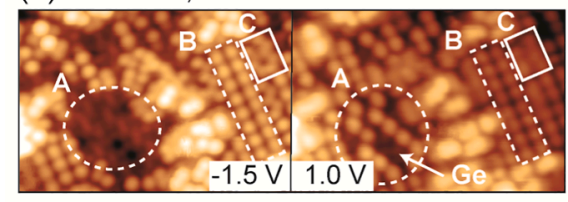

(f)

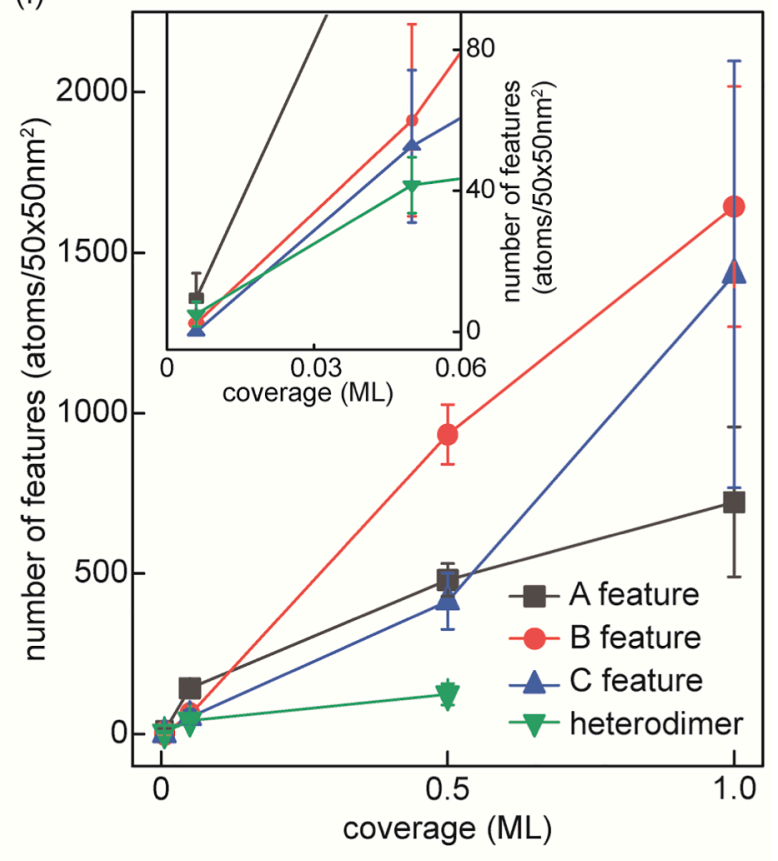

Fig. 5. Four different area coverages of $\mathrm{Sn}$ deposited on Ge(100). At submonolayer coverages (a-c) the surface reveals predominately the features discussed in Section 3.1 (i.e. A, B, C and heterodimer). At $1.24 \mathrm{ML}$ coverage (d) the A, B and C features form a closed layer (Layer 1). A second layer (Layer 2) has started to form with occasional adsorbents on a third layer (Layer 3). Clear distinction between the A, B, and C is shown in the close up STM image $\left(10 \times 15 \mathrm{~nm}^{2}\right)(\mathrm{g})$ taken in dual bias mode. Gaps in the small patches of ordered A features reveal the underlying Ge surface. The evolution of the heterodimer and A to $\mathrm{C}$ features as a function of coverage is plotted in (e). For the 1.24 ML sample only the features in Layer 1 are considered for the statistical analysis. All values are normalized to the available reactive sites and uncertainties arise from averaging over different areas measured on the same sample. surface, followed by feature $\mathrm{B}$, with feature $\mathrm{C}$ packing in the highest epitaxial density possible, corresponding to an additional atomic layer of the diamond-cubic configuration of the substrate below. As coverage increases, the number of atoms condensing in the $\mathrm{C}$ structure grows disproportionately faster than the lowest packing density A features, and to a lesser extent, the moderately dense B structure. For example, at $0.53 \mathrm{ML}$ coverage there are approximately twice as many B features on the surfaces as $C$ features, while at $1 \mathrm{ML}$ coverage their numbers are almost equal. This observation suggests a greater rate of capture and/or retention of atoms nucleating in the $\mathrm{C}$ structure compared to the lower density A and B structures, which appears counter-intuitive in terms of surface strain in the wetting layer as more ad-atoms become densely packed in layer 1 .

Overall, high resolution STM indicates that the RT deposition of Sn on $\mathrm{Ge}(001)$ results in the formation of a closed and atomically flat $\mathrm{Sn}$ wetting layer, with only minimal Ge intermixing (estimated $<2 \%$ of surface atoms) and no long-range ordering. We recall that at low submonolayer coverages the growth of ad-dimer chains aligned along the $\langle 310\rangle$ direction appears slightly favored, due to an additional diffusion barrier for ad-atoms to form $\langle 110\rangle$ dimer chains, and the A and B features are nearly equally present [19]. However, above 0.5 ML the amount of the energetically favored $\mathrm{B}$ and $\mathrm{C}$ features increases faster and the ratio between the $\mathrm{A}, \mathrm{B}$ and $\mathrm{C}$ features at 1.24 ML coverages deviates strongly from the low-coverage case studied by Tomatsu et al. [19]. The greater increase in the amount of B and C features compared to the A feature with increasing $\mathrm{Sn}$ coverage may be explained in terms of coverage dependent diffusion barriers. At higher coverages, an additional pathway of Sn diffusing on top of ad-dimer chains becomes available. The ad-atom can then adsorb on the surface at the end of the chain, elongating it. We propose that there is a lower barrier to diffusion along the top of the B features than on the A features, promoting B feature formation. As coverage increases, diffusion along $\mathrm{Sn}$ ad-dimers becomes more prevalent leading to a higher B feature contribution to the ad-dimers.

Finally, we wish to highlight the striking differences between the Ge/ $\mathrm{Si}$ and $\mathrm{Sn} / \mathrm{Ge}$ systems at full monolayer coverage. Typically grown at slightly elevated temperatures (e.g., $300{ }^{\circ} \mathrm{C}$ ), Ge forms an epitaxial wetting layer on $\mathrm{Si}(100)$ where the diamond-cubic structure of the substrate remains and strain is released through the presence of dimer vacancy lines perpendicular to the dimer rows and $\mathrm{Ge} / \mathrm{Si}$ intermixing $[26,27]$. In the $\mathrm{Sn} / \mathrm{Ge}$ wetting layer the only morphologically similar features are the patches of $C$ features, which make up only $37 \%$ of the surface. No dimer vacancy lines form, suggesting that the presence of multiple ad-dimer features reduces the strain in the wetting layer. Therefore, this strain relief mechanism prevents the formation of long range order. Moreover, in analogy to the Ge/Si system the observed Sn/ Ge intermixing at the surface may also contribute as a strain relief mechanism. The difference in surface morphology between the $\mathrm{Ge} / \mathrm{Si}$ 
and $\mathrm{Sn} / \mathrm{Ge}$ system can possibly be traced to the difference in chemical species involved. However, the reduced growth temperature utilized in this work, which leaves the system out of equilibrium, may also play a role. Yamazaki et al. [18] find that when growing 0.035 ML thick Sn layers on $\mathrm{Ge}$ at $250{ }^{\circ} \mathrm{C}$, the ad-dimer clusters characteristic of room temperature growth disappear. Instead the surface has a "comb-like" structure of long and thin terraces with the long direction being perpendicular to the dimer rows. This suggests, that if a Sn/Ge wetting layer was grown at elevated temperatures a surface more similar to the $\mathrm{Si} / \mathrm{Ge}$ system might be observed resulting in a surface with long range order. However, elevated growth temperatures may come at the cost of increased $\mathrm{Sn} / \mathrm{Ge}$ intermixing as the barrier to $\mathrm{Sn}$ incorporation becomes easier to overcome. Determining temperature best suited to minimize incorporation while maximizing long range order may be the subject of future studies.

\section{Conclusions}

In summary, we investigated the room temperature deposition of Sn on $\mathrm{Ge}(100)$ at low Sn coverage and the formation of a wetting layer at higher Sn coverages up to 1.24 ML. Upon adsorption of Sn on the Ge (100) surface, we reveal the two competing surface processes: (i) Sn incorporation into the top surface layer and (ii) the formation of addimer clusters. We investigate the chemical composition of the three types of ad-dimers that act as atomic-scale components that tile the surface as Sn coverage increases. By contrasting STM data with DFT, we show that $\mathrm{Ge}$ atoms ejected from the surface by $\mathrm{Sn}$ incorporation can also be present in the ad-dimer features.

When increasing the amount of Sn deposited, the ad-dimer clusters principally elongate perpendicular to the dimer rows, resulting in the growth of an atomically flat wetting layer. We observe is no long range order, and all three ad-dimer features (A, B and C) are still present. The completed wetting layer is dominated by the more densely packed $B$ and $\mathrm{C}$ features, whereas the lower density A features are at least equally represented at lower coverages. We propose that an increased Sn coverage changes the formation kinetics of these features, now favoring the $\mathrm{B}$ and $\mathrm{C}$ features. Furthermore, increasing the growth temperature slightly may allow for long range order to appear.

Due to $\mathrm{Sn}$ incorporation in the top Ge surface layer and resulting $\mathrm{Ge}$ ejection, the wetting layer presents a Sn dominated GeSn layer (estimated $<2 \% \mathrm{Ge}$ ), even at room temperature deposition. The $\mathrm{Ge} / \mathrm{Sn}$ intermixing during the early stages of Sn-on-Ge growth, limits the ultimate achievable sharpness of $\mathrm{Ge} / \mathrm{Sn}$ interfaces at the atomic level. Nonetheless, the two dimensionality of the layer formation at low growth temperatures makes this process well suited for fabrication of $\mathrm{Ge} / \mathrm{Sn}$ MQWs in future high performance Sn-based optical devices.

Overall, our results provide a first understanding of the complex growth mechanisms governing the transition from sub-monolayer to monolayer coverages in the $\mathrm{Sn} / \mathrm{Ge}$ and GeSn system. A next step could be the full chemical and electronic characterization of the wetting layer with techniques such as X-ray photoelectron spectroscopy (XPS) and angular resolved photoemission spectroscopy (ARPES). Interesting future STM studies may include the investigation of the growth mode of the second layer, subsequent higher coverages, and whether temperature variations can be used to further optimize the growth kinetics, e.g., in limiting the spread of $\mathrm{Sn}$ atoms in the out-of-plane direction.

\section{CRediT authorship contribution statement}

Emily V.S. Hofmann: Validation, Formal analysis, Investigation, Writing - original draft. Emilio Scalise: Methodology, Validation, Formal analysis, Writing - review \& editing. Francesco Montalenti: Conceptualization. Taylor J.Z. Stock: Supervision, Writing - review \& editing. Steven R. Schofield: Resources, Supervision, Writing - review \& editing. Giovanni Capellini: Conceptualization, Supervision, Writing - review \& editing. Leo Miglio: Conceptualization, Project administration, Supervision. Neil J. Curson: Conceptualization, Project administration, Funding acquisition, Supervision, Writing - review \& editing. Wolfgang M. Klesse: Conceptualization, Resources, Project administration, Funding acquisition, Supervision, Writing - review \& editing.

\section{Declaration of Competing Interest}

The authors declare that they have no known competing financial interests or personal relationships that could have appeared to influence the work reported in this paper.

\section{Acknowledgement}

This projected has been supported by the EPSRC project Atomically Deterministic Doping and Readout For Semiconductor Solotronics (grant number EP/M009564/1). E.V.S.H. is partly supported by the EPSRC Centre for Doctoral Training in Advanced Characterisation of Materials (grant number EP/L015277/1) and also IHP - Leibniz-Institut für innovative Mikroelektronik. Moreover, E.S. acknowledges the CINECA Consortium for the availability of high-performance computing resources and supports through the Italian SuperComputing Resource Allocation - ISCRA. E.V.S.H. would like to thank A. Becker for technical assistance at IHP.

\section{References}

[1] D. Lei, K.H. Lee, Y.-C. Huang, W. Wang, S. Masudy-Panah, S. Yadav, A. Kumar, Y. Dong, Y. Kang, S. Xu, Y. Wu, C.S. Tan, X. Gong, Y.-C. Yeo, Germanium-Tin (GeSn) P-Channel Fin Field-Effect Transistor Fabricated on a Novel GeSn-onInsulator Substrate, IEEE Trans. Electron Devices. 65 (2018) 3754-3761, https:// doi.org/10.1109/TED.2018.2856738.

[2] Y.-H. Huang, G.-E. Chang, H. Li, H.H. Cheng, Sn-based waveguide p-i-n photodetector with strained $\mathrm{GeSn} / \mathrm{Ge}$ multiple-quantum-well active layer, Opt. Lett. 42 (2017) 1652, https://doi.org/10.1364/OL.42.001652.

[3] A. Elbaz, D. Buca, N. von den Driesch, K. Pantzas, G. Patriarche, N. Zerounian, E. Herth, X. Checoury, S. Sauvage, I. Sagnes, A. Foti, R. Ossikovski, J.M. Hartmann, F. Boeuf, Z. Ikonic, P. Boucaud, D. Grützmacher, M. El Kurdi, Ultra-low-threshold continuous-wave and pulsed lasing in tensile-strained GeSn alloys, Nat. Photonics. (2020), https://doi.org/10.1038/s41566-020-0601-5.

[4] S. Wirths, R. Geiger, N. von den Driesch, G. Mussler, T. Stoica, S. Mantl, Z. Ikonic, M. Luysberg, S. Chiussi, J.M. Hartmann, H. Sigg, J. Faist, D. Buca, D. Grützmacher, Lasing in direct-bandgap GeSn alloy grown on Si, Nat. Photonics. 9 (2015) 88-92, https://doi.org/10.1038/nphoton.2014.321.

[5] J. Aubin, J.M. Hartmann, A. Gassenq, J.L. Rouviere, E. Robin, V. Delaye, D. Cooper, N. Mollard, V. Reboud, V. Calvo, Growth and structural properties of step-graded, high Sn content GeSn layers on Ge, Semicond. Sci. Technol. 32 (2017), 094006, https://doi.org/10.1088/1361-6641/aa8084.

[6] K.R. Khiangte, J.S. Rathore, V. Sharma, A. Laha, S. Mahapatra, Engineering strain relaxation of GeSn epilayers on $\mathrm{Ge} / \mathrm{Si}(001)$ substrates, Solid State Commun. 284-286 (2018) 88-92, https://doi.org/10.1016/J.SSC.2018.09.012.

[7] J. Chrétien, N. Pauc, F. Armand Pilon, M. Bertrand, Q.-M. Thai, L. Casiez, N. Bernier, H. Dansas, P. Gergaud, E. Delamadeleine, R. Khazaka, H. Sigg, J. Faist, A. Chelnokov, V. Reboud, J.-M. Hartmann, V. Calvo, GeSn Lasers Covering a Wide Wavelength Range Thanks to Uniaxial Tensile Strain, ACS Photonics. 6 (2019) 2462-2469, https://doi.org/10.1021/acsphotonics.9b00712.

[8] Y. Zhou, W. Dou, W. Du, S. Ojo, H. Tran, S.A. Ghetmiri, J. Liu, G. Sun, R. Soref, J. Margetis, J. Tolle, B. Li, Z. Chen, M. Mortazavi, S.Q. Yu, Optically Pumped GeSn Lasers Operating at $270 \mathrm{~K}$ with Broad Waveguide Structures on Si, ACS Photonics. 6 (2019) 1434-1441, https://doi.org/10.1021/acsphotonics.9b00030.

[9] F. Oliveira, I.A. Fischer, A. Benedetti, P. Zaumseil, M.F. Cerqueira, M.I. Vasilevskiy, S. Stefanov, S. Chiussi, J. Schulze, Fabrication of GeSn-multiple quantum wells by overgrowth of Sn on Ge by using molecular beam epitaxy, Appl. Phys. Lett. 107 (2015) 1-6, https://doi.org/10.1063/1.4938746.

[10] D. Rainko, Z. Ikonic, N. Vukmirović, D. Stange, N. von den Driesch, D. Grützmacher, D. Buca, Investigation of carrier confinement in direct bandgap GeSn/SiGeSn 2D and 0D heterostructures, Sci. Rep. 8 (2018) 15557, https://doi. org/10.1038/s41598-018-33820-1.

[11] P. Vogl, J. Olajos, W. Wegscheider, G. Abstreiter, Electronic structure and optical properties of short-period $\alpha$-SnnGem superlattices, Surf. Sci. 267 (1992) 83-86, https://doi.org/10.1016/0039-6028(92)91094-R.

[12] W. Wegscheider, J. Olajos, U. Menczigar, W. Dondl, G. Abstreiter, Fabrication and properties of epitaxially stabilized $\mathrm{Ge} / \alpha$-Sn heterostructures on $\mathrm{Ge}(001)$, J. Cryst. Growth. 123 (1992) 75-94, https://doi.org/10.1016/0022-0248(92)90012-8.

[13] K. Eberl, W. Wegscheider, G. Abstreiter, Group IV element (Si, Ge and $\alpha$-Sn) superlattices - low temperature MBE, J. Cryst. Growth. 111 (1991) 882-888, https://doi.org/10.1016/0022-0248(91)91101-F. 
[14] N.W. Hendrickx, D.P. Franke, A. Sammak, M. Kouwenhoven, D. Sabbagh, L. Yeoh, R. Li, M.L.V. Tagliaferri, M. Virgilio, G. Capellini, G. Scappucci, M. Veldhorst, Gatecontrolled quantum dots and superconductivity in planar germanium, Nat. Commun. 9 (2018) 1-7, https://doi.org/10.1038/s41467-018-05299-x.

[15] S. De Cesari, A. Balocchi, E. Vitiello, P. Jahandar, E. Grilli, T. Amand, X. Marie, M. Myronov, F. Pezzoli, Spin-coherent dynamics and carrier lifetime in strained Ge1-xSnx semiconductors on silicon, Phys. Rev. B. 99 (2019) 1-9, https://doi.org/ 10.1103/PhysRevB.99.035202.

[16] M. Liao, Y. Zang, Z. Guan, H. Li, Y. Gong, K. Zhu, X.-P.-P. Hu, D. Zhang, Y. Xu, Y.Y.-Y. Wang, K. He, X.-C.-C. Ma, S.-C.-C. Zhang, Q.-K.-K. Xue, Superconductivity in few-layer stanene, Nat. Phys. 14 (2018) 344-348, https://doi.org/10.1038/ s41567-017-0031-6.

[17] Y. Fang, Z.-Q. Huang, C.-H. Hsu, X. Li, Y. Xu, Y. Zhou, S. Wu, F.-C. Chuang, Z.Z. Zhu, Quantum Spin Hall States in Stanene/Ge(111), Sci. Rep. 5 (2015) 14196, https://doi.org/10.1038/srep14196.

[18] M. Yamazaki, S. Takeuchi, O. Nakatsuka, A. Sakai, M. Ogawa, S. Zaima, Scanning tunneling microscopy observation of initial growth of Sn and Ge1-xSnx layers on Ge(001) substrates, Appl. Surf. Sci. 254 (2008) 6048-6051, https://doi.org/ 10.1016/j.apsusc.2008.02.175.

[19] K. Tomatsu, K. Nakatsuji, T. Iimori, F. Komori, STM observation of initial growth of Sn atoms on Ge(001) surface, Surf. Sci. 601 (2007) 1736-1746, https://doi.org/ 10.1016/j.susc.2007.01.049.

[20] K. Tomatsu, K. Nakatsuji, T. Iimori, Y. Takagi, H. Kusuhara, A. Ishii, F. Komori, An Atomic Seesaw Switch Formed by Tilted Asymmetric Sn-Ge Dimers on a Ge (001) Surface, Science. 315 (2007) 1696-1698, https://doi.org/10.1126/ science.1137848.
[21] J.P. Perdew, K. Burke, M. Ernzerhof, Generalized Gradient Approximation Made Simple, Phys. Rev. Lett. 77 (1996) 3865-3868, https://doi.org/10.1103/ PhysRevLett.77.3865.

[22] P. Giannozzi, S. Baroni, N. Bonini, M. Calandra, R. Car, C. Cavazzoni, D. Ceresoli, G.L. Chiarotti, M. Cococcioni, I. Dabo, A. Dal Corso, S. de Gironcoli, S. Fabris, G. Fratesi, R. Gebauer, U. Gerstmann, C. Gougoussis, A. Kokalj, M. Lazzeri, L. Martin-Samos, N. Marzari, F. Mauri, R. Mazzarello, S. Paolini, A. Pasquarello, L. Paulatto, C. Sbraccia, S. Scandolo, G. Sclauzero, A.P. Seitsonen, A. Smogunov, P. Umari, R.M. Wentzcovitch, QUANTUM ESPRESSO: a modular and open-source software project for quantum simulations of materials, J. Phys. Condens. Matter. 21 (2009), 395502, https://doi.org/10.1088/0953-8984/21/39/395502.

[23] Y. Yoshimoto, Y. Nakamura, H. Kawai, M. Tsukada, M. Nakayama, Ge(001) surface reconstruction studied using a first-principles calculation and a Monte Carlo simulation, Phys. Rev. B. 61 (2000) 1965-1970, https://doi.org/10.1103/ PhysRevB.61.1965.

[24] X.R. Qin, M.G. Lagally, View of the empty states of the $\operatorname{Si}(100)-(2 \times 1)$ surface via scanning tunneling microscopy imaging at very low biases, Phys. Rev. B. 59 (1999) 7293-7296, https://doi.org/10.1103/PhysRevB.59.7293.

[25] G.A. Shah, M.W. Radny, P.V. Smith, S.R. Schofield, N.J. Curson, Electronic effects of single $\mathrm{H}$ atoms on $\mathrm{Ge}(001)$ revisited, J. Chem. Phys. 133 (2010), 014703, https://doi.org/10.1063/1.3456736.

[26] B. Voigtländer, M. Kästner, Evolution of the strain relaxation in a Ge layer on Si (001) by reconstruction and intermixing, Phys. Rev. B - Condens. Matter Mater. Phys. 60 (1999) R5121-R5124, https://doi.org/10.1103/PhysRevB.60.R5121.

[27] X. Chen, F. Wu, Z. Zhang, M.G. Lagally, Vacancy-vacancy interaction on Gecovered Si(001), Phys. Rev. Lett. 73 (1994) 850-853, https://doi.org/10.1103/ PhysRevLett.73.850. 